\title{
Simulations of density profiles, pellet fuelling and density control in ITER
}

\author{
L. Garzotti ${ }^{1}$, P. Belo ${ }^{2}$, G. Corrigan ${ }^{1}$, F. Köchl ${ }^{3}$, J. Lönnroth ${ }^{4}$, \\ V. Parail ${ }^{1}$, G. Pereverzev ${ }^{5}$, S. Saarelma ${ }^{1}$, G. Tardini ${ }^{5}$, M. \\ Valovič $^{1}$, I. Voitsekhovitch ${ }^{1}$, S. Wiesen ${ }^{6}$ \\ ${ }^{1}$ EURATOM-CCFE Association, Culham Science Centre, Abingdon, Oxon, OX14 \\ 3DB, United Kingdom \\ ${ }^{2}$ Associação EURATOM-IST, Instituto de Plasmas e Fusão Nuclear, Instituto \\ Superior Técnico, Av Rovisco Pais, 1049-001 Lisbon, Portugal \\ ${ }^{3}$ Association EURATOM-ÖAW/ATI, Atominstitut, TU Wien, 1020 Vienna, Austria \\ ${ }^{4}$ Helsinki University of Technology, Association EURATOM-Tekes, P.O.Box 4100, \\ FIN-02015 TKK, Finland \\ ${ }^{5}$ Max-Planck-Institut für Plasmaphysik, EURATOM-Assoziation, D-85748 Garching, \\ Germany \\ ${ }^{6}$ Forschungszentrum Jülich, Institute of Energy Research - Plasma Physics, \\ EURATOM Association, D-52425, Jülich, Germany
}

\begin{abstract}
The paper presents the results of 1.5-dimensional simulations of density profiles and pellet fuelling for the ITER baseline scenario performed with the ASTRA and JETTO transport codes by the ITER Scenario Modelling working group within the European Task Force on Integrated Tokamak Modelling. The first part of the paper describes the physics of the problem and how it is implemented in the different codes available to the working group. The second part presents the results of the simulations. Results obtained with the GLF23 physics based transport model and a simplified description of the pellet particle source are described alongside results obtained with the simpler Bohm/gyro-Bohm semi-empirical transport model and a more sophisticate pellet ablation/deposition code providing a completely self-consistent description of the pellet source. A parametric study has been performed to assess the effect of varying independently parameters, the values of which in ITER are either uncertain or not easily controllable (such as particle diffusivity, edge stability, wall recycling and boundary conditions), on the target plasma density, temperature, $Q$ and pellet frequency required to achieve a certain degree of density control. To this end the edge particle diffusivity was increased by a factor of three, the pedestal normalized critical pressure gradient for ballooning stability was decreased by $20 \%$, the boundary conditions on density and temperature were modified by $30-40 \%$ and the wall recycling particle source was increased from zero to $20 \%$ of the particle outflux. The results show that variations in the order of $15 \%$ for density and temperature, $40 \%$ for $Q$ and $100 \%$ for the pellet frequency can be expected. Open problems and modelling needs are also discussed in the paper.
\end{abstract}

PACS numbers: 52.55.Fa, 52.55.-s, 28.52.Cx, 52.25.Fi 


\section{Introduction}

In a thermonuclear plasma the deuterium-tritium fusion reaction rate, and consequently the energy produced, is proportional to the product between the densities of deuterium and tritium nuclei. To model a thermonuclear plasma like the one in ITER it is therefore important to simulate the behaviour of the density profile and the isotopic ratio. This problem is complicated because it has to take into account particle transport mechanisms in the plasma core, particle fluxes across the plasma separatrix and particle sources in the plasma due to either ionization of recycling atoms or additional fuelling methods like pellet injection and gas puffing (NBI fuelling being negligible in ITER).

Significant progress in understanding core particle transport has been made over the last few years, both from the experimental and theoretical point of view (for a recent overview of the subject and an extensive bibliography see, for example, [1] and references therein). This has led to the development of physics based transport models in reasonable agreement with experimental results $[2,3,4]$ and to a more convincing interpretation of data from present day machines $[5,6]$. On the other hand the problems of calculating the particle fluxes across the separatrix and the particle source due to neutral influx and pellet injection have not yet been completely solved.

The main problem in modelling particle fluxes through the plasma separatrix is that the assumptions underlying the transport models that describe reasonably well the particle transport in the core cease to be valid at the plasma periphery (the region called edge transport barrier or ETB in an H-mode plasma) and there are no reliable models to describe the particle flux in this part of the plasma. Moreover, in an H-mode plasma, like the one foreseen in ITER, intermittent edge instabilities called ELMs (edge localized modes) periodically expel particles from the plasma affecting therefore the plasma density. Models for the ELMs that are able to mimic the ELM bursts and can be coupled to a transport code are available (see, for example, [7]), but usually their predictive capability is limited because they depend on a number of free parameters that need to be adjusted to fit the experiments.

As for the description of the particle source, there are difficulties with the ionizing neutrals due to the fact that this phenomenon is intrinsically two-dimensional, involves complex atomic and molecular physics, depends on different space scales ranging from the atomic scale to the gradient scale at the plasma edge and implies the simultaneous description of two regions of the plasma (the ETB and the scrape-off layer or SOL) which have a completely different field line topology.

Concerning the fuelling, since the efficiency of gas puffing is likely to be insufficient to reach the required operational density, it is planned to fuel a thermonuclear plasma by means of frozen pellets of deuterium and tritium. The physics of the evaporation of cryogenic pellets is reasonably well understood and pellet ablation models have been developed and validated over the past thirty years (see, for example, [8] and references therein). However, a satisfactory and extensively validated description of the fast transport phenomena following the injection of a pellet in a thermonuclear plasma 
and therefore of the effective depth of the pellet particle source is still lacking and the extrapolation of present day experiments to ITER has to be taken with caution.

A final problem that has to be solved to model the evolution of the density profile is the prescription of the boundary conditions at the plasma separatrix. Strictly speaking, these can be provided by taking into account the full two-dimensional physics of the SOL. However, simulations of the SOL use as upstream boundary conditions particle and energy fluxes across the plasma separatrix and these depend in turn on the transport mechanisms in the plasma core. A way to solve this problem is to run a SOL code for a range of reasonable values for the fluxes across the separatrix and build a look-up table providing the appropriate boundary conditions corresponding to a particular level of core transport. A completely self-consistent solution of the problem would be obtained by using simultaneously a SOL and a core transport code coupled together. However, running such a code is extremely time consuming from a computational point of view.

This paper presents recent results of the modelling activity on ITER fuelling and density control performed by the ITER Scenario Modelling (ISM) working group [9] within the European Task Force on Integrated Tokamak Modelling. The scope of the work is to model in a way as complete and integrated as possible the density behaviour and the fuelling capabilities on ITER, taking in to account all the phenomena described above. The analysis concentrate on the ITER baseline scenario (sometimes referred to as ITER scenario 2), but from the point of view of density control and fuelling the results presented here can be applied to other ITER operating scenarios. The tools available inside the ISM for this kind of study were the two 1.5-dimensional core transport codes ASTRA [10] and JETTO [11] and the two-dimensional edge code EDGE2D [12], which can be run either as a stand alone code or integrated with JETTO in a complete coreedge transport code called JINTRAC. The results presented in this paper were obtained almost exclusively with the 1.5-dimensional transport codes ASTRA and JETTO and only an indirect use was made of the simulations done with JINTRAC. These will be extensively described in a future paper.

It should be noted that the codes mentioned above are not all equally well equipped to model particle transport and fuelling because they do not describe the underlying physics with the same degree of accuracy. Therefore we decided to start with simple simulations, gradually increase the level of complexity and deploy only the codes complex enough to deal with the problem in question.

The material will be presented in the following way: in section 2 we describe the different aspects of the physics of the problem and the way they are implemented in the various codes. Section 3 presents the results of the simulations of the steady-state density profiles. Section 4 introduces simple models for the ELMs and the fuelling. Section 5 will increase the degree of complexity of the description of these phenomena by introducing intermittent pellet injection and analyse the sensitivity of a particular fuelling scenario to plasma parameters such as particle diffusivity, edge stability, wall recycling and boundary conditions, of which we have either limited control or little idea of their actual value on ITER. A discussion of the results will be given in section 6 and 
conclusions in section 7 .

\section{Models and codes for the physics of density evolution and control}

\subsection{Core transport}

All codes deployed in the ISM working group are equipped with a number of models to describe core particle and heat transport. The first type of models is based on scaling laws to derive formulae for particle diffusivity $D$ and electron and ion thermal conductivity $\chi_{i}$ and $\chi_{e}$ and ensure that the transport coefficients are dynamically adjusted with the plasma parameters to keep the energy confinement time in agreement with the ITER-98(y,2) confinement scaling, as described in [9]. The second type of models are semi-empirical models like the Bohm/gyro-Bohm transport model [14]. The third type of models are physics based models like the GLF23 [2], Weiland [3],or MultiMode transport models [13]. For the study presented in this paper we have adopted the Bohm/gyro-Bohm and the GLF23 transport model.

The implementation of GLF23 in ASTRA and JETTO adopts a numerical scheme to avoid instabilities described in [15], hence allowing longer time steps in the integration of the transport equation and faster runs in terms of CPU consumption. However, the advantages of this implementation are limited to steady-state simulations. When used to model plasmas with intermittent fuelling and density profiles rapidly oscillating from peaked to hollow (a situation far from the one for which it was tuned), GLF23 started to exhibit numerical instabilities induced by the appearance of regions where the density gradient is close to zero.

For this reason, when an intermittent pellet model, which induces strong transients in the simulations, was deployed, we reverted to the more robust semi-empirical Bohm/gyro-Bohm model. However, it should be noted that this model contains a number of free parameters that were tuned to simulate JET discharges as described in [14]. In the ITER simulations presented in this paper the values of these parameters were modified to obtain average density and temperature compatible with $Q \approx 10$ as expected in a typical ITER baseline scenario. In practice, with respect to the JET case, the Bohm contribution to the electron and ion thermal conductivity was enhanced by a factor of four, whereas the gyro-Bohm contribution was enhanced by a factor of eight and sixteen respectively. Moreover, to allow for a peaked density profile as the one predicted by GLF23, an inward particle pinch velocity was added to the conventional diffusive term describing the particle flux in the model.

\subsection{Edge physics}

As mentioned in the introduction, the core transport models described in the previous subsection are not adequate to model the transport inside the transport barrier at the edge of a typical H-mode plasma. Moreover, to model the plasma edge it is necessary to simulate the ELMs that appear after the L-H transition and affect the transport at 
the plasma periphery. This situation can be modelled in two ways. The simplest is to dynamically adjust the transport inside the ETB in order to maintain the pressure gradient just below the threshold at which the instability responsible for the ELM is triggered. This description is called the continuous ELM model. In practice the transport coefficient $D$ and $\chi_{e, i}$ inside the ETB are calculated as:

$$
\begin{aligned}
& D=D^{n c}+C_{1} \cdot \max \left(0, \frac{\alpha_{\max }}{\alpha_{c}}-1\right)^{\beta} \\
& \chi_{e, i}=\chi_{e, i}^{n c}+C_{2,3} \cdot \max \left(0, \frac{\alpha_{\max }}{\alpha_{c}}-1\right)^{\beta}
\end{aligned}
$$

where $D^{n c}$ is the neo-classical particle diffusivity, $\chi_{e, i}^{n c}$ are the electron and ion neoclassical thermal conductivities, $C_{1-3}$ are constants (in our case $C_{1}=C_{2}=C_{3}=100$ $\left.\mathrm{m}^{2} / \mathrm{s}\right), \alpha_{\max }$ is the maximum normalized pressure gradient inside the ETB and $\alpha_{c}$ is the normalized critical pressure gradient at which the ETB becomes ballooning unstable. The latter value is determined according to MHD stability calculations. Finally, $\beta$ is a constant exponent (in our simulations $\beta=2$ ). This model is implemented in all the codes used in this study, however, the actual values of $\alpha_{c}$ may vary from code to code due to different normalization of the pressure gradients. In particular, in JETTO, the normalized pressure gradient $\alpha$ is defined as:

$$
\alpha=3.125 \mu_{0}\left(R_{\text {out }}(\rho)-R_{\text {out }}(\rho=0)\right)^{2}\left|\frac{\partial p_{e}(\rho) / \partial \rho+\partial p_{i}(\rho) / \partial \rho}{\partial \Psi / \partial \rho B_{\text {pol }, \text { out }}(\rho)}\right|
$$

where $\mu_{0}$, is the vacuum permeability, $R_{\text {out }}$ is the major radius of the flux surface at the outer mid-plane, $p_{e}$ and $p_{i}$ are the thermal pressures for electrons and ions, $\Psi$ is the poloidal flux, $B_{\text {pol,out }}$ is the poloidal magnetic field strength at the outer midplane, $\rho=\sqrt{\Phi / \pi B_{\text {ref }}}$, where $\Phi$ is the toroidal flux, $B_{\text {ref }}$ is the vacuum magnetic field at a reference position for the geometrical centre of the vacuum chamber (in JETTO $R_{\text {ref }}=6.2 \mathrm{~m}$ is usually prescribed for ITER and $R_{\text {ref }}=2.95 \mathrm{~m}$ for JET). With this definition of $\alpha$, the value for $\alpha_{c}$ was set to 1.7 .

The definition of $\alpha$ in ASTRA is:

$$
\alpha=-2 R_{0} \mu_{0} \partial p(\rho) / \partial \rho\left(\frac{q}{B}\right)^{2}
$$

where $R_{0}$ is the plasma geometric major radius, $p$ the plasma pressure, $q$ the safety factor, $B$ the toroidal magnetic field and $\rho$ the square root of the normalized toroidal magnetic flux. To be consistent with the JETTO simulation and taking into account the different definition of $\alpha$, the value of $\alpha_{c}$ in ASTRA was set to 2 .

A second way to deal with ELMs is the intermittent ELM model. In this case the transport inside the ETB is assumed to be very low (e. g. neo-classical) and pressure gradient inside the ETB is allowed to increase until it reaches a critical value. At this point the transport coefficients are increased (typically by a factor of 100) for a fixed time $(\sim 1 \mathrm{~ms})$ during which the pressure gradient relaxes below the critical value [16]. This model simulates better the intermittent nature of the ELMs but is likely to introduce 
numerical instabilities especially when coupled with less robust transport models for the core like GLF23.

\subsection{Sources and fuelling}

The particle source due to the ionization of the neutral atoms recycling at the wall is described in JETTO by FRANTIC [17], where a ray tracing technique is used to calculate the neutral density inside the plasma in cylindrical geometry, and in ASTRA by directly solving the kinetic equation for the distribution function of the neutral atoms in slab geometry [10]. In both cases ionization and multiple charge exchange processes are taken into account to determine the profile of the recycling particle source. The strength of the source is calculated by imposing that the volume integral of the source is equal to a given fraction of the particle flux leaving the plasma. It is worth nothing that both modules are one-dimensional and cannot describe the poloidal asymmetries of the neutral density profile.

The description of the particle source due to pellet injection requires the simultaneous modelling of three phenomena: the evaporation of the pellet material, the parallel expansion of this material along the magnetic field line and the fast $\nabla B$ induced drift that displaces the ablation cloud towards the low field side of the tokamak leading from the initial ablation profile to the final deposition profile [18]. Among the codes available for this study only JETTO is equipped with modules to describe the pellet particle source. In fact, JETTO is equipped with a neutral gas and plasma shield (NGPS) ablation module [19] and a more complete ablation/deposition module, called HPI2 [20]. Both codes calculate the intermittent pellet particle source self-consistently with the target plasma parameters. The first code deals with the physics of the pellet evaporation and the expansion of the pellet cloud along the magnetic field lines and calculates the pellet evaporation and the pellet source profile without taking into account the fast $\nabla B$ drift displacing the pellet material towards the low field side of the tokamak. This code is fast and can be used either when the effect of the drift is not dramatic or by artificially enhancing the pellet injection speed to obtain the desired depth for the pellet particle source (although in this case the consistence with the target plasma parameters is lost). The second code takes into account, on top of the pellet ablation and cloud expansion, also the full effect of the $\nabla B$ drift to provide a fully self-consistent description of the pellet particle source. This code is much slower and can be used when only few pellets need to be simulated or when computation time is not an issue and a high degree of approximation for the shape of the pellet particle source is required.

Other codes can use the results of these modules to simulate the pellet source either in a continuous or an intermittent way. The simpler option is the continuous pellet source, whereby a particle source is added to the transport equations with a radial profile modelled on the pellet deposition profile and an intensity tuned to match the actual time-averaged fuelling due to the intermittent pellet injection. This option has the advantage of simulating the pellet particle source in a smooth continuous way and is 
less likely to induce numerical instability in the integration of the transport equations. A more realistic implementation of the same concept can be achieved by switching on the pellet source for a duration corresponding to the pellet ablation time and tune its intensity to match the total pellet particle input as before. A limit of this approach is that the pellet source profile is prescribed and not modelled self-consistently with the evolving kinetic profiles.

An important point to keep in mind when using an intermittent particle source like pellet injection is the large perturbation induced in the particle and energy fluxes across the separatrix. These oscillations over a pellet injection cycle cause a variation of the density and temperature profiles in the SOL, which in turn influence the boundary conditions at the separatrix and other typical parameters of the SOL plasma near the divertor strike points, like the fraction of radiated power and the degree of detachment. As mentioned before a full solution of this problem requires the integration of a core and an edge transport code and is beyond the scope of this paper.

\section{Modelling of the steady-state gas-fuelled density profile}

The starting point of the analysis of density behaviour in an ITER baseline scenario was the simulation of the target steady-state density profile. This was achieved with ASTRA and JETTO equipped with the GLF23 transport model. All the simulations presented in the following are fully predictive, in the sense that equations are solved for the plasma current density, deuterium and tritium density, ion and electron temperature. The electron density and the impurity concentration were calculated consistently with the prescribed $Z_{\text {eff }}$ profile and the evolution of rotation was not considered.

Before performing fully predictive simulation for ITER, we benchmarked ASTRA and JETTO against each other to test the implementation of GLF23 in the two codes and validated the results against JET shot 61109. This deuterium shot, together with similar ones, is described in [21] and was chosen because it has relatively low collisionality $\left(\nu^{*} \approx 0.015\right)$, exhibits a peaked density profile due to an anomalous pinch velocity and therefore represents a suitable starting point for extrapolation to ITER. ASTRA and JETTO were set up in order to match the boundary conditions at $\sqrt{\phi_{N}}=0.85$ (where $\phi_{N}$ is the normalized toroidal flux) and run until a steady state was reached. This is because the GLF23 transport model becomes unreliable in the pedestal and therefore it was decided not to model this region of the plasma.

The NBI particle source and power deposition profiles were calculated using the PENCIL module [22] available in JETTO and the same profiles were used in ASTRA. This is true in general for all the simulations performed in this study using the ASTRA and JETTO codes. The consequence of this choice is that while, on the one hand, similar simulations share the same NBI particle and energy sources, on the other hand the source profiles are consistent with the density and temperature profiles in JETTO but not in ASTRA. However, since the ASTRA and JETTO simulated kinetic profiles are always very similar to each other, this should not constitute a problem. 
The results of the simulations were compared with the experimental density profiles measured by the JET LIDAR Thomson scattering system and averaged over a five seconds time window, during which the density could be considered stationary. The results are illustrated in figure 1 and, for $\sqrt{\phi_{N}} \leq 0.85$, show good agreement between the two codes and with the experimental data.

The simulation of an ITER baseline scenario are shown in figure 2. As in the JET case the boundary conditions were matched at $\sqrt{\phi_{N}}=0.85$ and the codes run until a steady state was reached. A peaking factor (defined as the ratio between the density at $\sqrt{\phi_{N}}=0.2$ and the volume average density $\left.\left\langle n_{e}\right\rangle\right)$ of 1.3 is found. This value is in line with extrapolations based on the analysis of a large multi-machine database of density profiles, which gives values for the density peaking factor between 1.3 and 1.4 [6], and another study based on a smaller database of JET plasmas, which gives a value between 1.2 and $1.3[5]$.

In both JET and ITER simulations a steady-state density profile was obtained by imposing a recycling factor $R=1$. This is equivalent to assuming that all the ions leaving the plasma reenter as neutrals, or, alternatively, that gas puffing can replace exactly the particles of fuel that leave the plasma and are pumped from the vessel.

\section{Steady-state modelling of the pellet source}

A first simulation of the effect of pellet fuelling on the steady-state density profiles was performed with the ASTRA transport code. The analysis is similar to the one presented in [23]. In this case ASTRA was run in combination with the GLF23 transport model. Continuous ELMs and continuous pellets models were applied inside the ETB. The boundary conditions were imposed at the separatrix and adapted in order to have $n_{e}=0.8 \cdot 10^{20} \mathrm{~m}^{-3}$ and $T_{e}=T_{i}=6 \mathrm{keV}$ at top of the barrier. $Z_{\text {eff }}$ was assumed constant with radius and equal to 1.7 . In the simulations a particle source profile simulating pellet injection was artificially introduced in the transport equations. The pellet particle source profile was obtained by running the HPI2 ablation code to calculate the deposition profile of a typical ITER fuelling pellet (cubic $5 \mathrm{~mm}$ side, particle content $7.5 \cdot 10^{21}$ atoms), injected in an ITER scenario 2 plasma with ITER-like high field side (HFS) injection geometry. The source intensity was tuned to simulate a pellet injection frequency of $4.6 \mathrm{~Hz}$, corresponding to a net particle flux of $3.5 \cdot 10^{22}$ atoms/s and the fact that in this case gas puffing was replaced with pellet injection was simulated by setting the recycling factor $R=0$.

The results are shown in figure 3 . Frames a) and b) show the electron density and temperature profiles obtained when the steady state is reached. Frames c) and d) represent the effective particle and electron thermal diffusivities given by GLF23 in combination with the continuous ELMs model. Frame e) shows the normalized pellet particle source profile. It can be seen that, under this fuelling regime and with the transport coefficients shown in the figure, a peaked electron density profile with on axis density of $1.4 \cdot 10^{20} \mathrm{~m}^{-3}$ can be sustained. Corresponding on-axis temperature are 
$T_{e}=17.7 \mathrm{keV}$ and $T_{i}=14.6 \mathrm{keV}$. Under this conditions the plasma performances are characterized by fusion power $P_{\text {fus }}=360 \mathrm{MW}$ corresponding to $Q=11$. The same simulation was performed with JETTO and, despite some differences between the two codes (e. g. in the way the equilibrium is calculated), almost identical results in terms of profiles, $P_{\text {fus }}$ and $Q$ were obtained.

\section{Intermittent modelling of the pellet source}

In order to increase the degree of complexity of the simulations and to investigate the effect of different plasma parameters on the pellet injection requirements, we used JETTO, which, at the moment, is the only transport code at our disposal coupled with a full ablation/deposition code. As mentioned in section 2.1, due to problems of reliability with the GLF23 transport model when used in combination with hollow density profiles and an intermittent particle source, in this kind of simulations, we had to reduce the degree of complexity with which the transport was treated by employing the numerically more robust Bohm/gyro-Bohm model. The $D$ and $V$ profiles used in the simulations are shown in figure 4. A feedback mechanism for pellet injection was implemented so that a pellet was injected every time the density at the top of the ETB dropped below $7.5 \cdot 10^{19} \mathrm{~m}^{-3}$. Auxiliary power was $33 \mathrm{MW}$ NBI. Boundary condition were imposed at the separatrix and were set to $n_{e}=4 \cdot 10^{19} \mathrm{~m}^{-3}, T_{e}=550 \mathrm{eV} T_{i}=850$ $\mathrm{eV}$. The continuous ELM model was used with $\alpha_{c}=1.7$ to create an ETB and clamp the value of the pressure gradient inside it just below the ballooning instability limit. Under these conditions the simulations relaxed towards a quasi-steady-state regime with characteristic density and temperature profiles and $Q$.

The effect of different depth for pellet particle source due to different displacements induced by the $\nabla B$ drift was discussed in [9]. In that study three runs were performed, one with the full displacement provided by the ablation/deposition code and providing a pellet particle source profile peaking at $\sqrt{\phi_{N}} \approx 0.92$, one where the displacement was reduced by $50 \%$ and the pellet particle source profile peaked at $\sqrt{\phi_{N}} \approx 0.95$, and one without any displacement with the pellet particle source profile peaking at $\sqrt{\phi_{N}} \approx 0.97$. The main result of the analysis was that for the three penetration depths the pellet injection frequency required to maintain a quasi-steady-state density profile stabilized at 1,2 and $5 \mathrm{~Hz}$ respectively.

To expand the analysis and to investigate the effect of other parameters on the ITER fuelling scenario more simulations were performed. These simulations are described in the following subsections and the results are summarized in table 1.

\subsection{Case 1: base case}

The base case for this detailed study was similar to the $50 \%$ displacement fully predictive simulation described in [9] and the results are shown in figure 5 and 6 . It can be seen that, after an initial transient, the on-axis density stabilizes at $1.3 \cdot 10^{20} \mathrm{~m}^{-3}$ the volume 
average density at $1.0 \cdot 10^{20} \mathrm{~m}^{-3}$, the on-axis electron temperature at $15 \mathrm{keV}$ and the volume average electron temperature at $8 \mathrm{keV}$. The oscillations in the density at the top of the barrier induced by the injection of a pellet when its value drops below $7.5 \cdot 10^{19}$ $\mathrm{m}^{-3}$ can be clearly seen from the figure. Likewise the edge plasma cooling caused by the pellet is clearly seen as oscillation of the electron temperature at the top of the barrier. When a quasi steady-state situation is reached the number of electrons crossing the separatrix per unit time oscillates during a pellet injection cycle between $1.1 \cdot 10^{22}$ $\mathrm{s}^{-1}$ and $2.5 \cdot 10^{22} \mathrm{~s}^{-1}$ and around an average value over a pellet cycle of $1.5 \cdot 10^{22} \mathrm{~s}^{-1}$. The pellet frequency for this particular case stabilizes at $2.4 \mathrm{~Hz}$ and the $Q$ value at 12 .

\subsection{Case 2: increased particle diffusivity in the pellet ablation region}

Since the density and temperature perturbations induced by the pellets are likely to increase the level of turbulence and hence transport in the region where the ablation occurs [24], we decided to asses the effect of an increased particle diffusivity with respect to the one predicted by the Bohm/gyro-Bohm transport model. In practice the particle diffusion coefficient was enhanced in the region affected by the pellet ablation by a factor of three with respect to the base scenario. The factor three is justified by the experimental observation that the density perturbation induced by the pellet relaxes on a faster time scale than predicted by the Bohm/gyro-Bohm transport model and that the experimental post-pellet evolution of the density profile can be simulated by enhancing the particle diffusivity by a factor of three in the Bohm/gyro-Bohm model [25]. The results of this simulations are presented in figure 7 and 8. As a result of the increased diffusivity the on-axis density stabilized at a lower value, with respect to the reference case, of $1.1 \cdot 10^{20} \mathrm{~m}^{-3}$ and the volume average density at $8.5 \cdot 10^{19} \mathrm{~m}^{-3}$. As a consequence of the reduced density the fusion power is reduced and this results in lower steady-state value for the temperature as well. For this run the on-axis electron temperature stabilizes at $13 \mathrm{keV}$, but since this drop takes place in a relatively small volume in the plasma core the volume average electron temperature is only marginally reduced to $7.5 \mathrm{keV}$. The particle losses across the separatrix oscillate between $6 \cdot 10^{21} \mathrm{~s}^{-1}$ and $8 \cdot 10^{21} \mathrm{~s}^{-1}$ around an average value of $7 \cdot 10^{21} \mathrm{~s}^{-1}$. The pellet frequency necessary to maintain this lower density steady state is $1.2 \mathrm{~Hz}$ and the steady-state $Q$ settles at around 7 .

The fact that both particle losses across the separatrix and pellet frequency settle to a lower value when the diffusivity is increased over the pellet ablation region is counter intuitive and deserves some comment. Indeed, this is a consequence of the way we treat the ETB in the simulations and can be understood as follows: the increased diffusivity inside the edge transport barrier results in a less peaked density profile. This in turn implies a reduction in alpha heating, a lower temperature in the plasma core and a lower thermal flux at the top of the ETB. Since in our model for the ETB both density and temperature gradients are kept constant inside the ETB because of the feedback mechanism on the density at the top of the ETB and of $\alpha_{c}$ clamping the pressure gradient 
inside the ETB, the only way to accommodate a reduced thermal flux across the ETB is to reduce $\chi_{i, e}$ inside the ETB. As a consequence of the fact that in the continuous ELM model the ratio $D / \chi$ is kept constant inside the ETB also $D$ is reduced in this region. This implies a reduction in the particle outflux through the separatrix, a slower decay of the density at the top of the ETB and, eventually, because of the feedback mechanism controlling the pellet injection, a lower pellet injection frequency necessary to maintain the quasi-equilibrium density.

It is clear that although the reduction in the quasi-steady-state density and temperature is a robust result, the predicted reduction in particle outflux and pellet frequency is very sensitive to the model used to describe the transport within the ETB and should be taken with caution because the model adopted in our simulations is extremely simplified and, although it is effective in keeping the value of $\alpha$ within the ETB close to the limit imposed by MHD stability, it does not capture the details of the transport mechanisms in this region. However, this model retains some physical meaning since a lower plasma pressure resulting from a reduced fusion power source would probably lead to a lower ELM frequency and therefore lower particle losses induced by the ELMs.

\subsection{Case 3: decreased critical pressure gradient for edge MHD stability}

The second test we performed was aimed at assessing the effect of the ELMs and of the general edge MHD stability on the fuelling performances. This test was prompted by the finding that the threshold for ballooning instability in ITER might be lower than the one assumed to estimate the gradient inside the ETB [16]. To do this we decreased the value of $\alpha_{c}$ from 1.7 to 1.4 in the continuous ELM model, to simulate the effect of a lower ballooning stability threshold for the plasma edge. The results are shown in figure 9 and 10. It can be seen that the density behaviour does not change much, whereas the on-axis electron temperature drops to $12.5 \mathrm{keV}$, the electron temperature at the top of the ETB decreases on average by $1 \mathrm{keV}$ with respect to the reference case, the average electron temperature stabilizes at $7 \mathrm{keV}$ and $Q$ decreases to 9 . This is a consequence of the fact that because of the nature of the density feedback mechanisms we are keeping the density gradient inside the ETB fixed. By reducing the value of the pressure gradient we are essentially reducing the temperature gradient in the ETB. Since the width of the ETB is constant in our simulations, this results in a lower temperature pedestal and therefore, for the same transport model in a lower on-axis and volume average electron temperature and a lower $Q$. In this case we found that the pellet frequency is decreased to $1.9 \mathrm{~Hz}$ and the particle losses are similar to the reference case. This is not surprising since the density profile and the particle diffusivity are unchanged from the reference case. 


\subsection{Case4: simulations with finite recycling}

The third test was done to assess the influence of the wall recycling particle source on the fuelling scenario. The simulations described so far were done with no particle influx from the wall. To quantify the sensitivity of the fuelling performances to this parameter we increased the fraction of wall recycling neutrals to $20 \%$. The results, shown in figure 11 and 12, indicate that as a consequence of the increased particle source the required pellet frequency decreased by $10 \%$, the density and temperature profile, net particle outflux and $Q$ were practically unchanged with respect to the reference case. This is easy to understand and is due to the fact that the additional source represented by the ions recycling on the wall helps sustain the density at the top of the ETB and therefore less frequent pellets are necessary to keep it above the chosen value of $7.5 \cdot 10^{19} \mathrm{~m}^{-2}$.

\subsection{Case 5: modified boundary conditions at the separatrix}

The last test involved the boundary conditions imposed to the simulation. Until now we have imposed the boundary conditions without taking into consideration the effect on the density at the separatrix and in the SOL of the additional particle outflux associated with the pellet injection. This task, strictly speaking, can only be achieved by running simultaneously a core transport code and an edge transport code. This is an extremely time consuming analysis and represents a long term program outside the scope of this paper. However, some separate investigation was done by running the edge code EDGE2D and varying the number of particle crossing the separatrix from $2 \cdot 10^{21} \mathrm{~s}^{-1}$ (representative of a situation where the pellet is ablated deeper in the plasma) to $10^{23} \mathrm{~s}^{-1}$ (corresponding to a very peripheral ablation of the pellet). With increasing particle throughput, the electron and ion densities at the separatrix calculated by EDGE2D increased from 4 to $8 \cdot 10^{19} \mathrm{~m}^{-3}$, whereas the electron and ion temperatures at the separatrix decreased from 550 to $350 \mathrm{eV}$ and from 850 to $450 \mathrm{eV}$ respectively.

To analyse the effect of varying boundary conditions a simulation was performed with main ion density at the separatrix increased from 4 to $5.3 \cdot 10^{19} \mathrm{~m}^{-3}$ and electron and ion temperature at the separatrix decreased from 800 to $500 \mathrm{eV}$. The results are shown in figure 13 and 14. By increasing the density at the separatrix and maintaining the density at top of the barrier fixed we decrease the density gradient within the ETB. The results is a decrease in particle losses from between $1.1 \cdot 10^{22} \mathrm{~s}^{-1}$ and $2.5 \cdot 10^{22} \mathrm{~s}^{-1}$ to between $1.5 \cdot 10^{21} \mathrm{~s}^{-1}$ and $1.4 \cdot 10^{22} \mathrm{~s}^{-1}$. With this reduced particle losses the pellet frequency necessary to maintain the density at the top of the ETB at $7.5 \cdot 10^{19} \mathrm{~m}^{-3}$ decreased from $2.4 \mathrm{~Hz}$ to $0.4 \mathrm{~Hz}$. This decreased pellet frequency results in a lower on-axis and volume average density of $12 \cdot 10^{19} \mathrm{~m}^{-3}$ and $9 \cdot 10^{19} \mathrm{~m}^{-3}$ respectively and a lower value of $Q$ of 9 .

To conclude this part of the analysis it should be noted that on one side EDGE2D predicts higher densities at the separatrix with higher particle flux across the plasma boundary, whereas JETTO predicts lower particle losses with higher density at the separatrix. The reason for the two different trends is that JETTO prescribes the density 
at the separatrix and controls the density at the top of the ETB, whereas EDGE2D does not take into account the density at the top of the ETB and only calculates the density at the separatrix once the flux at the top of the ETB is prescribed. An equilibrium point where the two codes give the same density and temperature at the separatrix must exist and to determine this point a fully integrated and self consistent analysis of this problem, including the effect of pellet fuelling on the detachment of the divertor is needed. This analysis is ongoing and will be presented in a future paper.

\section{Discussion}

The simulations described in the previous paragraphs illustrate what can be done, in terms of modelling with 1.5-dimensional transport codes and with different degrees of complexity, to predict the fuelling requirements in the ITER baseline scenario. They also show the limits of this approach and the uncertainties to be expected when a particular fuelling scenario will be implemented on ITER. In this paragraph we point out these limitations and indicate what should be done to overcome them and what implications they have from the practical point of view of designing a fuelling scheme for a reactor plasma.

From the modelling point of view it is clear that the results depend critically on the models adopted for the transport both in the core and the ETB and for the ELMs. In the simulations presented we have used either a physics based transport model (GLF23) or a semi-empirical model with a limited number of free parameters (Bohm/gyro-Bohm). GLF23 has proved numerically unstable when the shape of density profile oscillates between peaked and hollow due to intermittent pellet injection. Moreover, this model is not valid in the region of the plasma $r / a \gtrsim 0.8$. Therefore results of transport simulations must be taken with caution. Finally, no reliable model for the ELMs is available and therefore the expulsion of fuel following the injection of a pellet (and the effect of a pellet on the ELM cycle) cannot be modelled with accuracy. This shows the necessity of developing, on the one side, transport models that can be applied to the outer region of the plasma and in presence of oscillating plasma profiles and, on the other side, reliable predictive models for the ELM induced losses. In particular, a useful model to describe the effect of the ELMs on the fuelling should be interfaceable with a 1.5-dimensional transport code and predict, on the basis of the plasma profiles, when an ELM is triggered and what the post ELM profiles will be.

A second problem from the point of view of the simulation is related to the boundary conditions. Strictly speaking this problem can be treated self consistently by coupling a 1.5-dimensional transport code for the plasma core and a two-dimensional transport code for the SOL, acting in an integrated fashion. Alternatively, an approach similar to the one adopted in the integrated core pedestal SOL (ICPS) model described in [26] could be considered. In this case scaling laws deduced from a database of two-dimensional SOL simulations are used to assure consistency of operating conditions between the core and the SOL. It is important to note, however, that, when treating intermittent pellet 
injection, consistency will have to be enforced dynamically during the entire pellet cycle (i. e. density increase and subsequent profile relaxation) and not only in a steady-state situation.

Another important point highlighted by the simulations presented in the previous paragraphs is the conditions under which to request pellet fuelling. Pellet injection could be pre-set at a given frequency in order to achieve a fixed equilibrium density. However, this will give no control on the plasma performance if the confinement property of the discharge change in time. Alternatively a feed-back scheme could be implemented. Assuming that the pellet frequency can be varied, in this study we have implemented a system aiming at keeping the density at the top of the ETB above a certain value. However, as we have seen, with different target plasma this does not guarantee that the fusion power and therefore the $Q$ factor remains the same. Another possibility (not considered in the present study) would be to feed-back directly on the fusion power output or on the neutron production rate, as proposed in [27], or on some quantity more representative of the central density like the line average plasma density. More analysis of the optimization of the fuelling scheme and density control mechanism in terms of sensors and actuators is needed from the control point of view.

\section{Conclusions}

In this paper advanced simulations of density behaviour and control in ITER performed in the framework of the ISM working group within the European Task Force on Integrated Tokamak Modelling were presented. Simulations performed with the physics based GLF23 transport model and where intermittent ELMs and pellet injection were replaced by continuous processes showed that in the ITER baseline scenario $Q \geq 10$ is achievable and peaked density profiles (peaking factor $\approx 1.3$ ) can be sustained and fuelled with a pellet particle source of $3.5 \cdot 10^{22}$ particle/s, corresponding to a pellet injection frequency of $4.6 \mathrm{~Hz}$ if the pellet mass is $7.5 \cdot 10^{21}$ atoms. Clearly this fuelling scenario would result in a burden for the pumping system of exactly $3.5 \cdot 10^{22}$ particle/s. This corresponds to a pumping speed of $\approx 65 \mathrm{~Pa} \mathrm{~m}^{3}$, which is below the maximum pumping speed of $120 \mathrm{~Pa} \mathrm{~m}^{3}$ presently in the ITER design. It should be noted, however, that these results, although corroborated by the fact that they were obtained with two different codes (ASTRA and JETTO), cannot be easily extrapolated to the case of a more realistic intermittent particle source and oscillating density and temperature profiles, where GLF23 does not provide a reliable description of the transport.

Further simulations were performed with the JETTO transport code where a realistic description of the intermittent pellet particle source was implemented. However, due to the numerical problems that emerged when GLF23 was used in combination with zero density gradients, to analyse this situation we had to revert to the semiempirical Bohm/gyro-Bohm transport model. This second set of simulations allowed us to analyse the impact on the fuelling performances and the final quasi steady-state plasma parameters of the uncertainties in the reaction of the particle diffusivity to 
the perturbation induced by the pellet, the marginally stable pressure gradient in the plasma ETB, the boundary conditions at the separatrix compatible with a fully detached divertor and the influence of the wall recycling source.

It was shown that varying independently each parameter can result in a maximum variation of the pellet frequency required to maintain a certain density at the top of the ETB of at least a factor of two, whereas the variation of average density and temperature is in the order of $10-15 \%$ and of $Q$ in the order of $40 \%$. A value of $Q \geq 7$ seems to be achievable in all the cases analysed.

The simulations showed also the limits of the description we can provide with the models presently available and indicated that a better description of particle transport in presence of hollow density profiles, a reliable transport model applicable in the ETB and a good description of the ELMs and of the reaction of the ELM cycle to the pellet fuelling are necessary.

Finally we showed that, if a feed-back scheme is to be implemented to control the pellet fuelling system, a dedicated study will have to be performed to determine the parameter (or combination of parameters) one will have to feedback on.

\section{Acknowledgments}

This work was funded partly by the RCUK Energy Programme under grant EP/I501045 and the European Communities under the contract of Association between EURATOM and CCFE. The views and opinions expressed herein do not necessarily reflect those of the European Commission. This work was carried out within the framework of the European Fusion Development Agreement.

\section{References}

[1] Angioni C, Fable E, Greenwald M, Maslov M, Peeters A G, Takenaga H and Weisen H 2009 Plasma Phys. Control. Fusion 51124017

[2] Waltz R E, Staebler G M, Dorland W, Hammett G W, Kotschenreuther K and Konings J A 1997 Phys. Plasmas 42482

[3] Strand P, Nordman H, Weiland J and Christiansen J P 1998 Nucl. Fusion 38545

[4] Weiland J 2000 Collective Modes in Inhomogeneous Plasma - Kinetic and Advanced Fluid Theory (Bristol and Philadelphia: Institute of Physics Publishing)

[5] Valovič M, Garzotti L, Voitsekhovitch I, Beurskens M, Garbet X, Giroud C, Keeling D and JET EFDA contributors 2007 Nucl. Fusion $\mathbf{4 7} 196$

[6] Angioni C et al 2009 Nucl. Fusion 471326

[7] Lonnröth J S, Parail V, Dnestrovskij A, Figarella C, Garbet X and Wilson H 2004 Plasma Phys. Control. Fusion 461197

[8] Pégourié B 2007 Plasma Phys. Control. Fusion 49 R87

[9] Parail V et al 2009 Nucl. Fusion 49075030

[10] Pereverzev G V and Yushmanov P N 2002 ASTRA Automated System for TRansport Analysis in a Tokamak, Max-Planck-Institut fur Plasmaphysik, IPP-Report 5/98

[11] Cenacchi G Taroni A 1988 JETTO: A free-boundary plasma transport code (basic version), JET Joint Undertaking, Report JET-IR(88)03

[12] Simonini R, Corrigan G, Radford G, Spence J and Taroni A 1994 Contrib. Plasma Phys. 34368 
[13] Bateman G, Kritz A H, Kinsey J E, Redd A J and Weiland J 1998 Phys. Plasmas 51793

[14] Erba M, Cherubini A, Parail V V, Springmann E and Taroni A 1997 Plasma Phys. Control. Fusion 39261

[15] Pereverzev G V and Corrigan G 2008 Comput. Phys. Comm. 179579

[16] Lonnröth J, Parail V, McDonald D C, Saarelma S, de La Luna E, Beurskens M and JET EFDA contributors 2011 Nucl. Fusion 51013003

[17] Tamor S 1981 J. Comput. Phys. 40104

[18] Rozhansky V, Veselova I and Voskoboynikov S 1995 Plasma Phys. Control. Fusion 37399

[19] Garzotti L, Pégourié B, Géraud A, Frigione D and Baylor L R 1997 Nucl. Fusion 371167

[20] Pégourié B, Waller V, Nehme H, Garzotti L and Géraud A 2007 Nucl. Fusion 4744

[21] Valovič M et al 2004 Plasma Phys. Control. Fusion 461877

[22] Challis C. D. et al 1989 Nucl. Fusion 29563

[23] Kamelander G, Weimann G, Garzotti L, Litaudon X, Moreau D, Pégourié B 2004 Fusion Sci. Tech. $\mathbf{4 5} 558$

[24] Valovič M et al 2008 Nucl. Fusion 48075006

[25] Garzotti L, Corrigan G, Heading D, Jones T T C, Parail V, Pégourié B and Spence J 2000 27th European Physical Society Conf. on Controlled Fusion and Plasma Physics Contributed Papers (Budapest, Hungary, 12-16 June 2000) vol 24B (ECA) eds K. Szegö et al (Mulhouse: European Physical Society) 316

[26] Pacher G W, Pacher H D, Janeschitz G and Kukushkin A S 2008 Nucl. Fusion 48105003

[27] Moreau D, Voitsekhovitch I 1999 Nucl. Fusion 39685 
Table 1. Summary of the simulation results described in section 5. (Intermittent modelling of the pellet source, Bohm/gyro-Bohm transport model and continuous ELM model in the ETB).

\begin{tabular}{|c|c|c|c|c|c|c|c|c|}
\hline Case & Description & $\begin{array}{c}n_{e}(0) \\
{\left[10^{19} \mathrm{~m}^{-3}\right]}\end{array}$ & $\begin{array}{c}\left\langle n_{e}\right\rangle \\
{\left[10^{19} \mathrm{~m}^{-3}\right]}\end{array}$ & $\begin{array}{l}T_{e}(0) \\
{[\mathrm{keV}]}\end{array}$ & $\begin{array}{l}\left\langle T_{e}\right\rangle \\
{[\mathrm{keV}]}\end{array}$ & $Q$ & $\begin{array}{c}f_{p} \\
{[\mathrm{~Hz}]}\end{array}$ & $\begin{array}{c}\left\langle\Gamma_{\text {out }}\right\rangle \\
{\left[10^{22} \mathrm{~s}^{-1}\right]}\end{array}$ \\
\hline 1 & Reference case & 13 & 10 & 15 & 8 & 12 & 2.4 & 1.5 \\
\hline 2 & Enhanced particle diffusivity & 11 & 8.5 & 13 & 7.5 & 7 & 1.2 & 0.7 \\
\hline 3 & Normalized critical pressure gradient $\alpha_{c}=1.4$ & 14 & 11 & 12.5 & 7 & 9 & 1.9 & 1.2 \\
\hline 4 & Recycling $R=0.2$ & 13 & 10 & 15 & 8 & 12 & 2.2 & 1.5 \\
\hline 5 & Modified boundary conditions & 12 & 9 & 15 & 8 & 9 & 0.4 & 0.2 \\
\hline
\end{tabular}




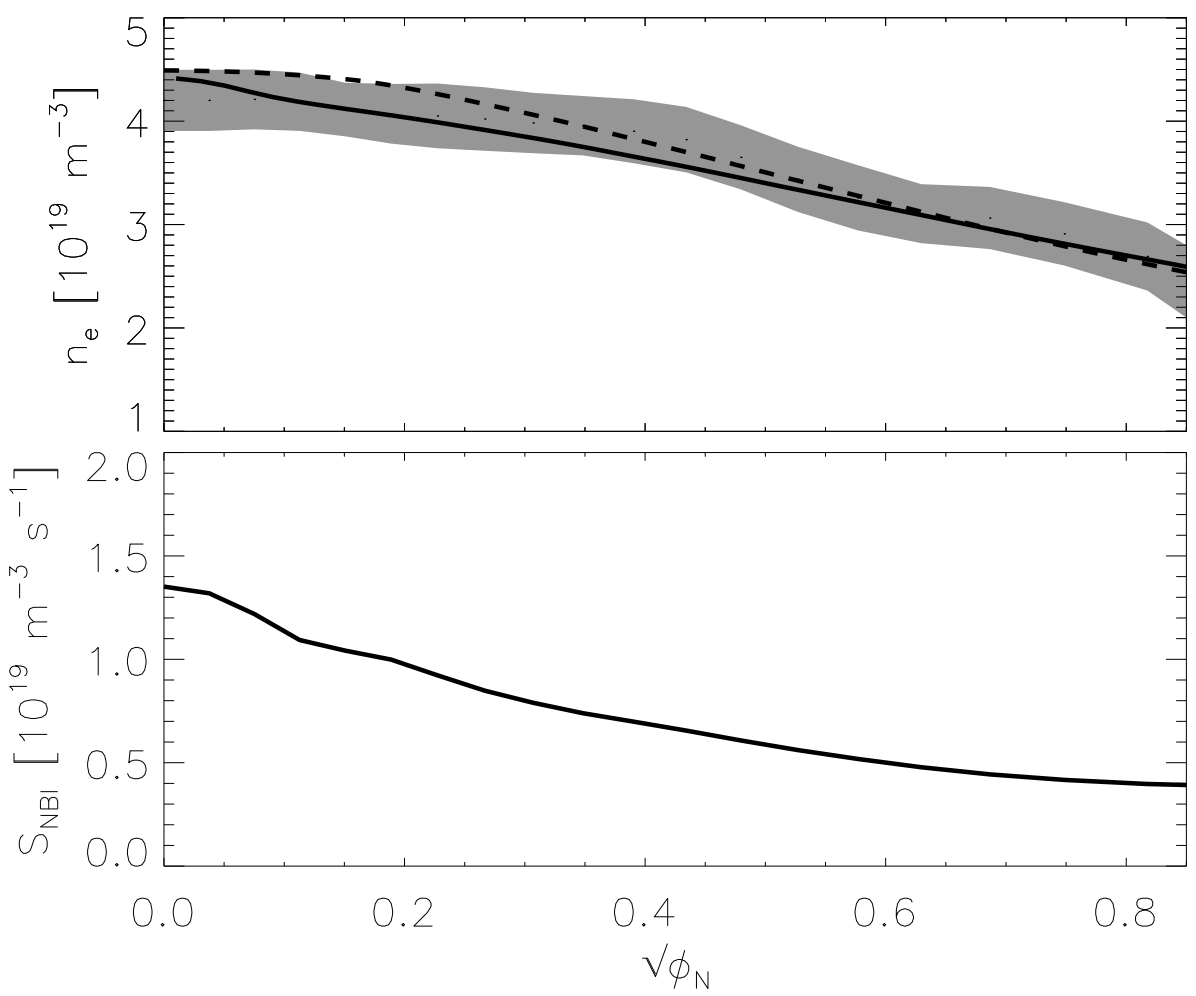

Figure 1. Simulation of JET shot 61109. Top: electron density profile from ASTRA (dashed line) and JETTO (solid line), both equipped with the GLF23 transport model. The shaded area is the experimental profile with associated error bars. Bottom: NBI particle source used in the simulation. 

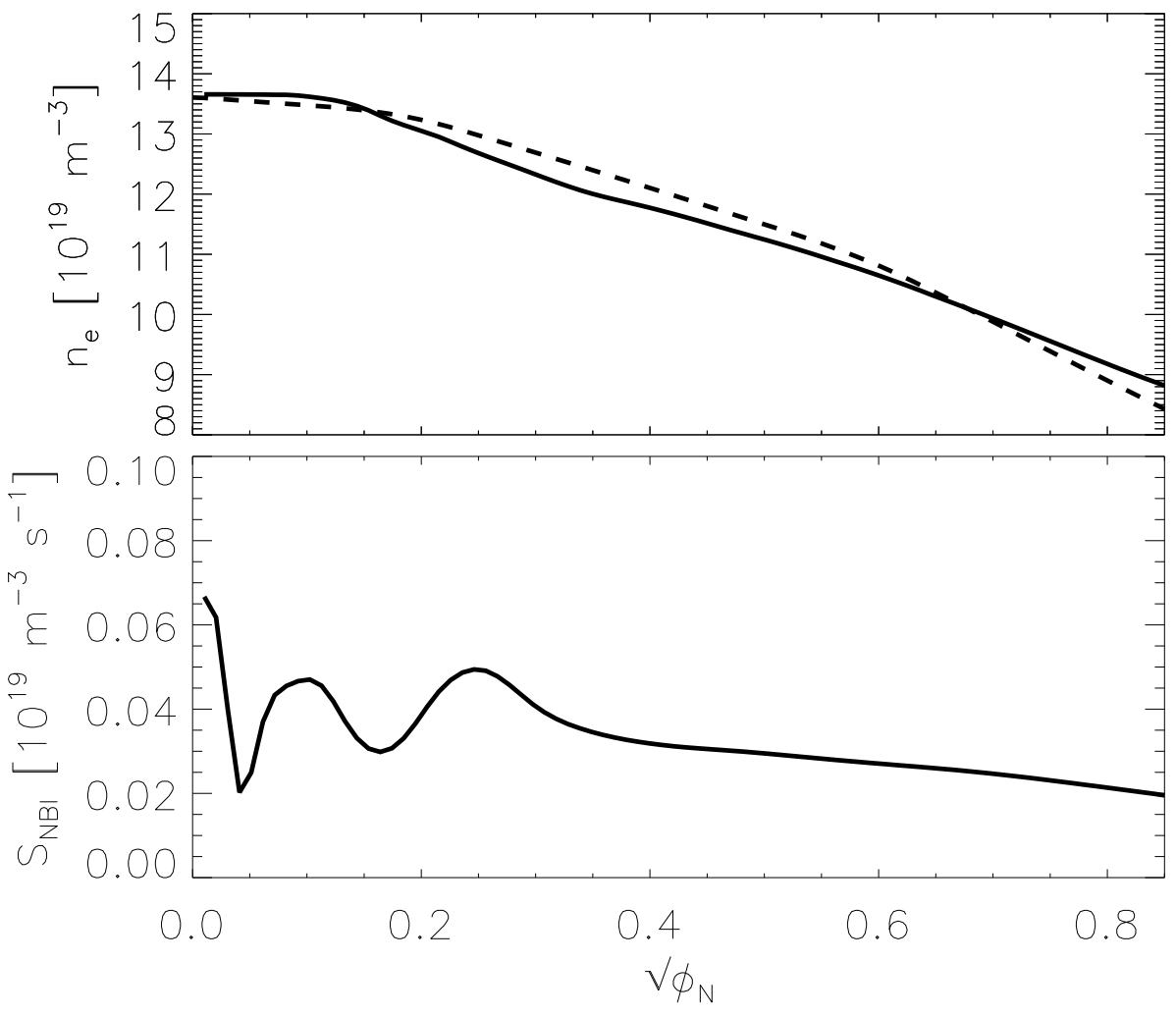

Figure 2. Simulation of ITER scenario 2. Top: density profile from ASTRA (dashed line) and JETTO (solid line), all equipped with the GLF23 transport model. Bottom: NBI particle source used in the simulation. The two peaks in the particle source profile are due to the ITER neutral beam injection geometry with one beam lying on the machine equatorial plane and the other tilted with respect to it. 

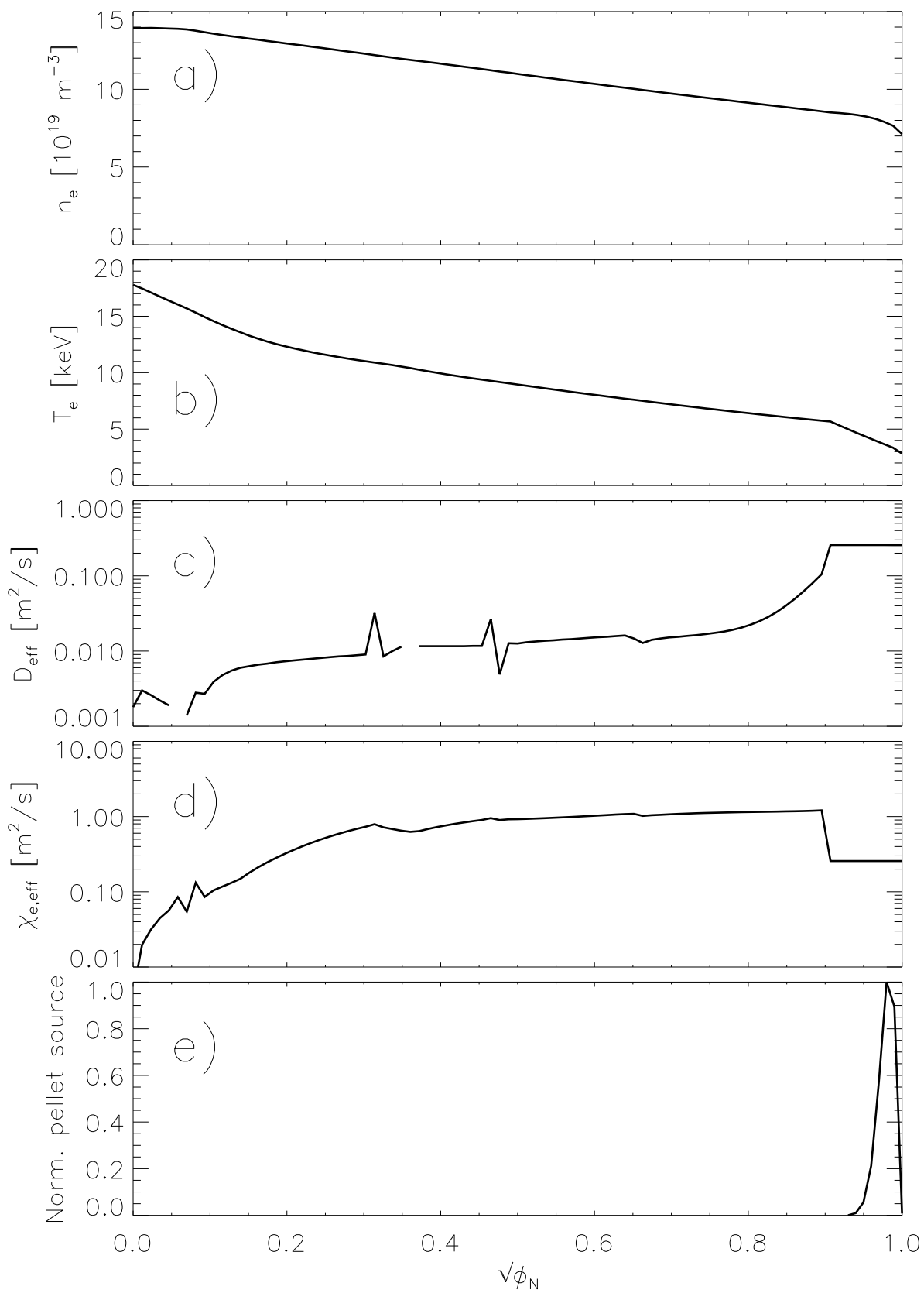

Figure 3. Simulation of a pellet fuelled ITER scenario 2 plasma with ASTRA equipped with the GLF23 transport model, continuous ELMs and continuous pellets. a) electron density profile, b) electron temperature profile, c) effective particle diffusivity, d) effective electron thermal conductivity, e) normalized pellet particle source. 

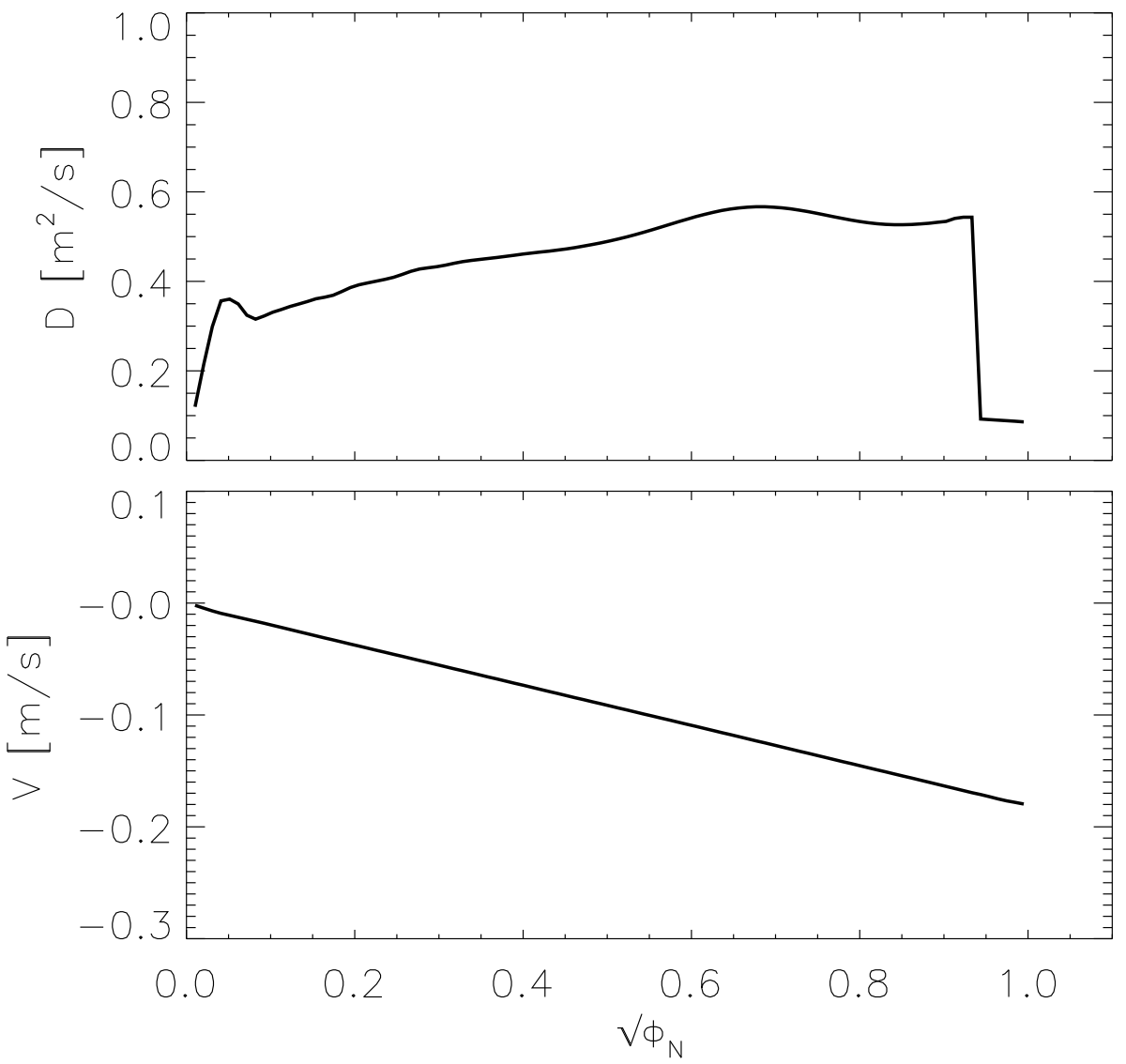

Figure 4. Radial profiles of the particle diffusivity (top) and convective velocity (bottom) used in the JETTO simulations of the ITER pellet fuelled baseline scenario. 

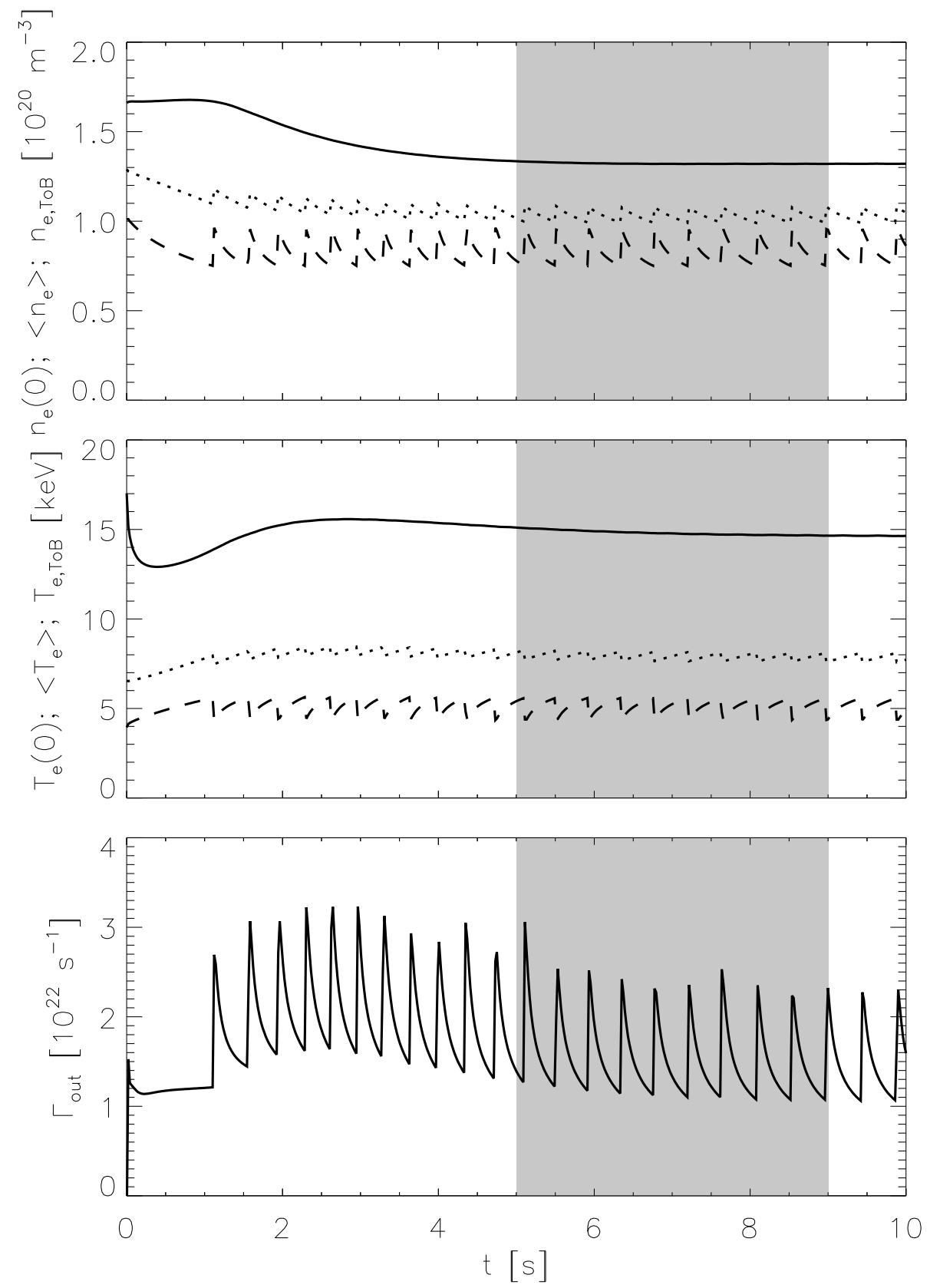

Figure 5. Evolution of plasma density (top) temperature (middle) and particle losses (bottom) for the reference simulation of the ITER pellet fuelled baseline scenario. In the top and middle frames solid lines correspond to on-axis values, dotted lines to volume averages and dashed lines to values at the top of the edge transport barrier. The grey shaded area highlights the time window where the quantities can be considered quasi stationary and averaged. 

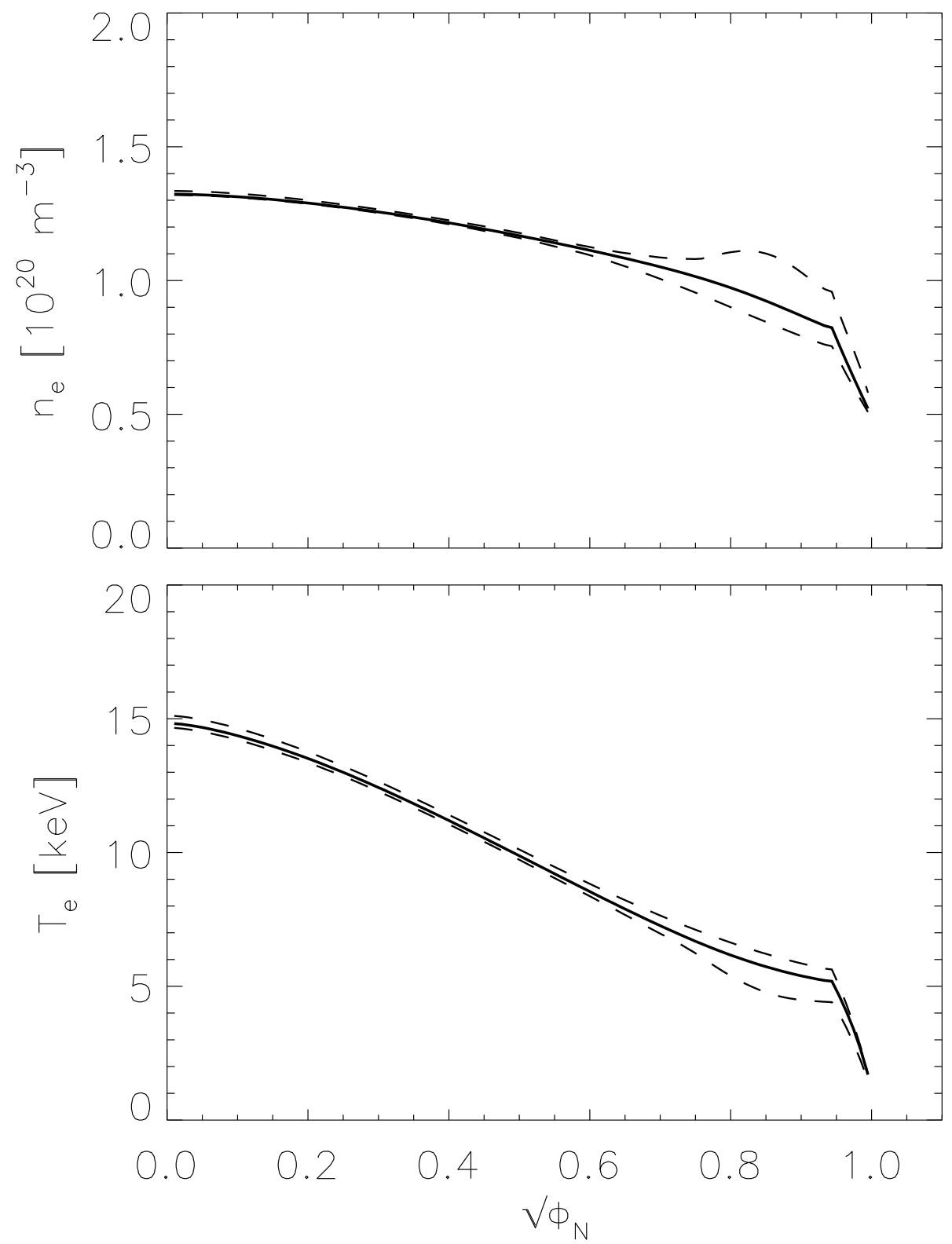

Figure 6. Electron density (top) and temperature profiles for the reference simulation of the ITER pellet fuelled baseline scenario. Solid lines indicate profiles averaged over the time window highlighted in 5 and dashed lines show the extremes between which the profiles oscillate between two successive pellet injections. 

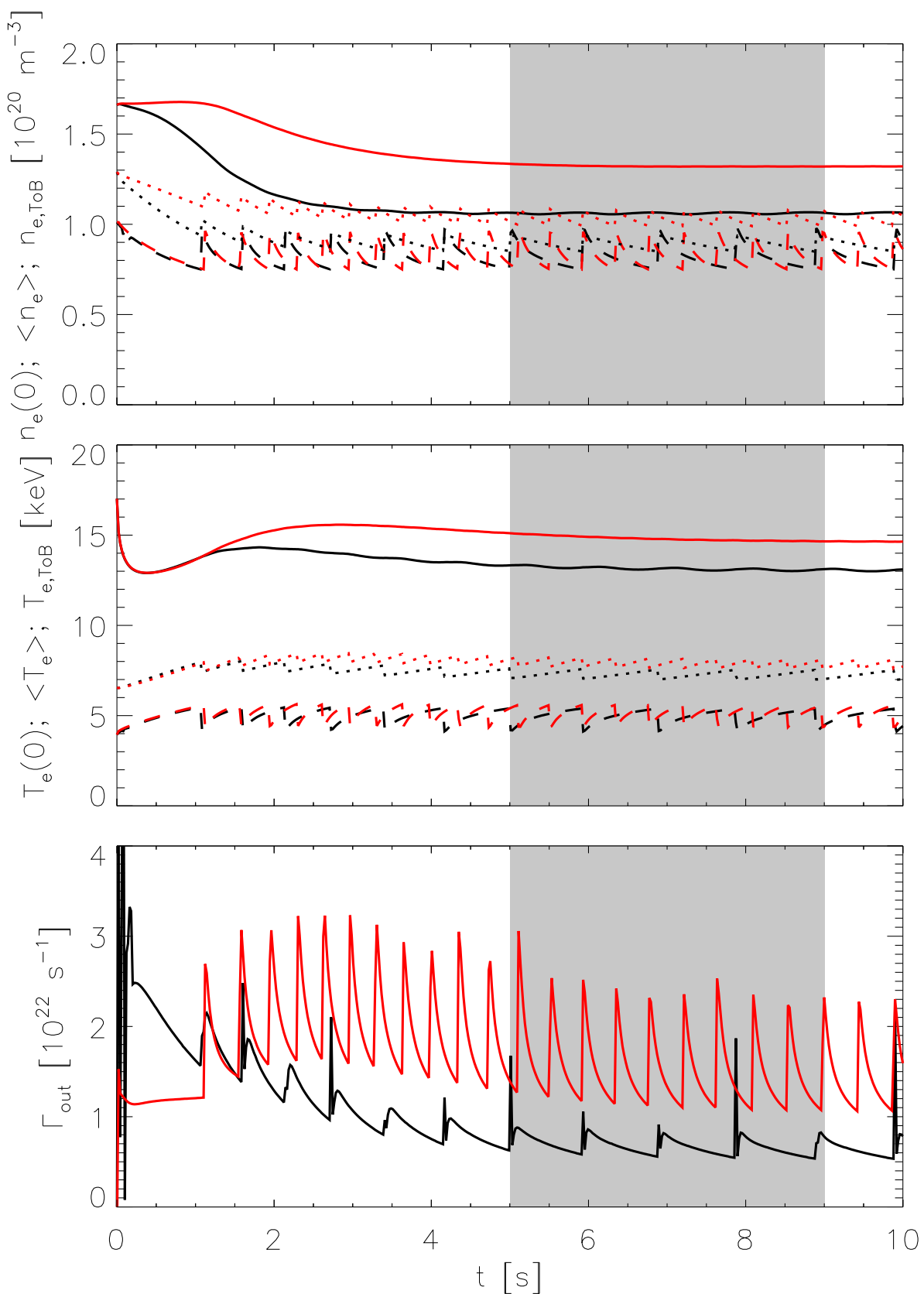

Figure 7. Same as figure 5 for the case where the Bohm/gyro-Bohm particle diffusivity was increased by a factor of three in the region affected by the pellet ablation. The red lines correspond to the base case and are shown for comparison. 

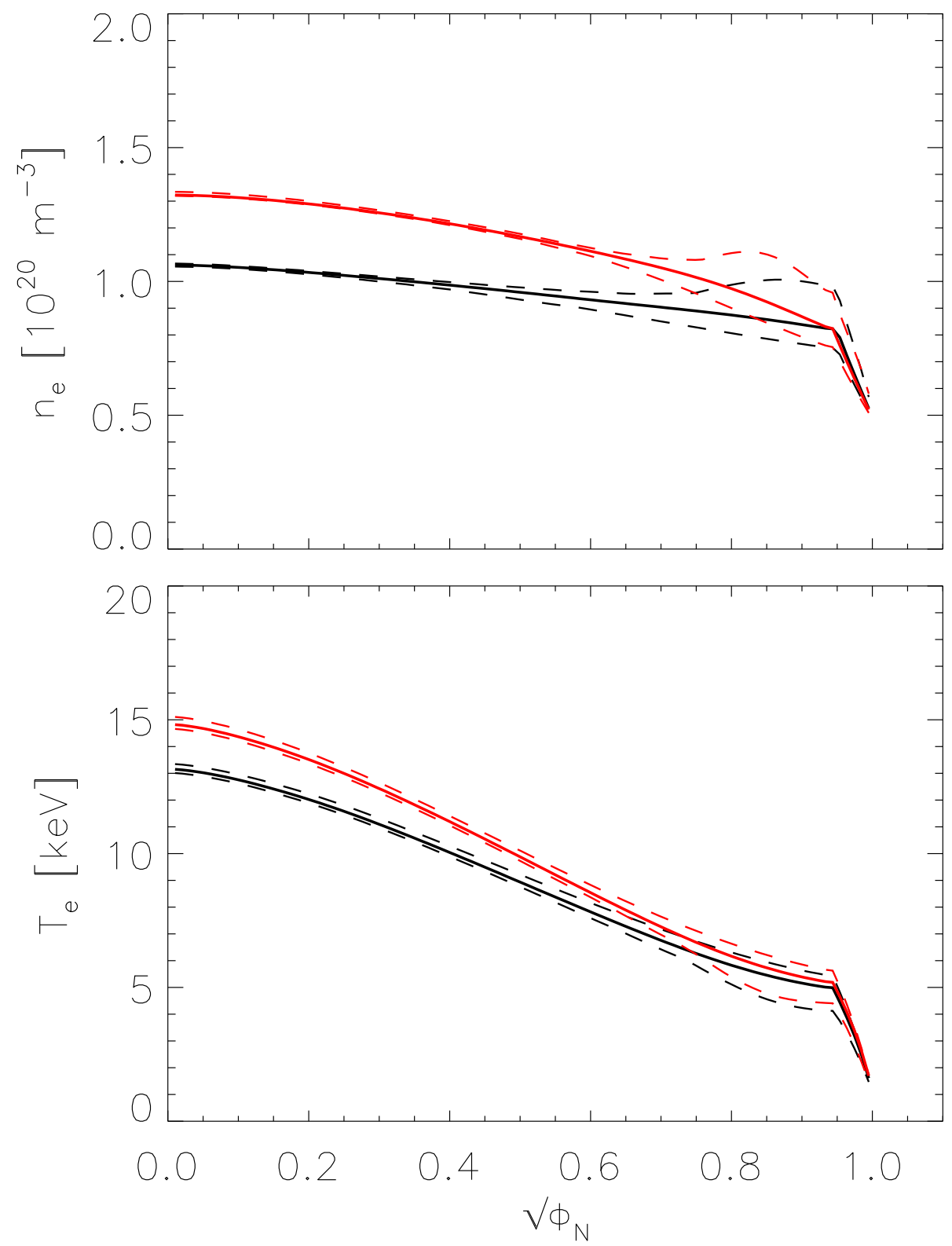

Figure 8. Same as figure 6 for the case where the Bohm/gyro-Bohm particle diffusivity was increased by a factor of three in the region affected by the pellet ablation. The red lines correspond to the base case and are shown for comparison. 

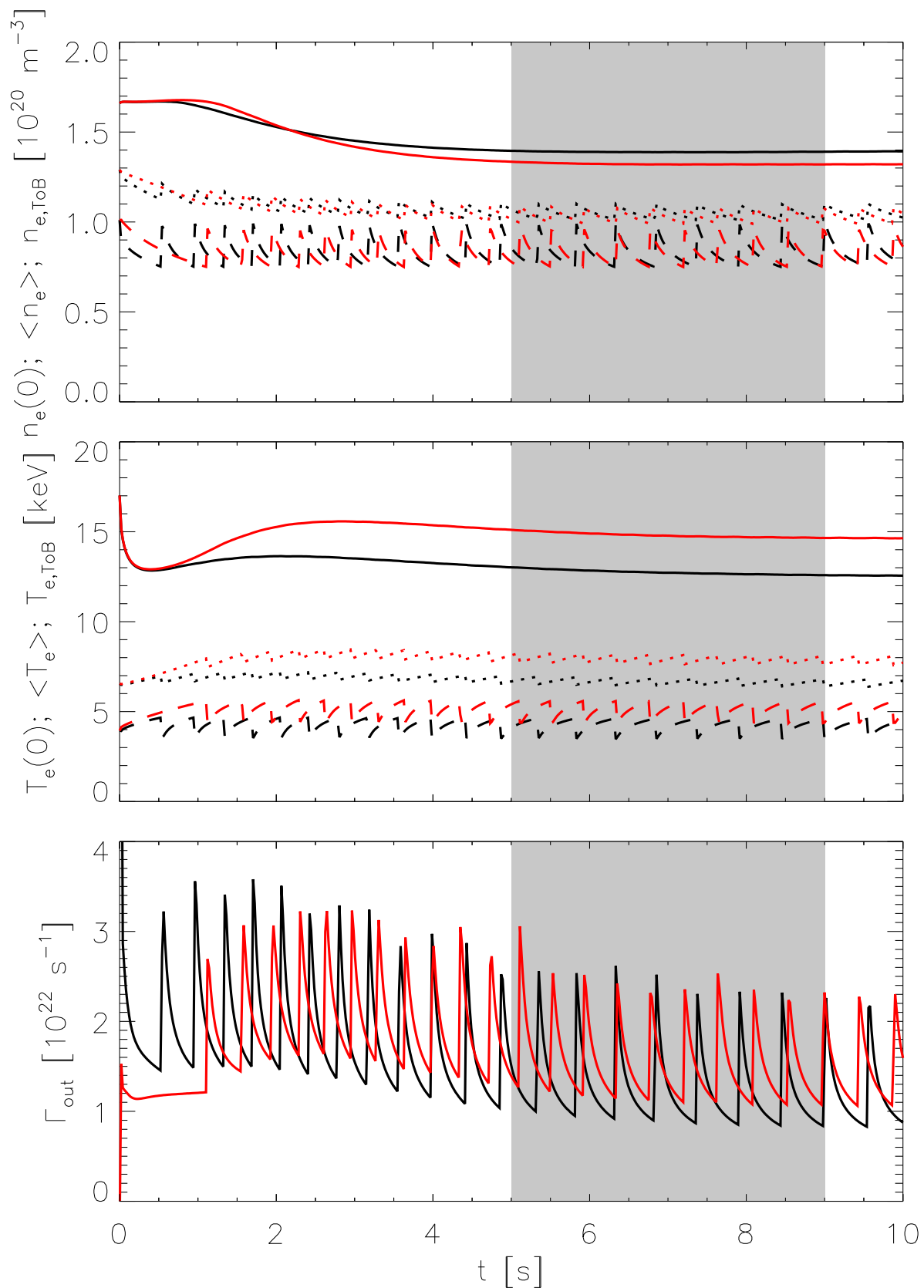

Figure 9. Same as figure 7 for the case where $\alpha_{c}$ in the continuous ELM model was reduced from 1.7 to 1.4 . 

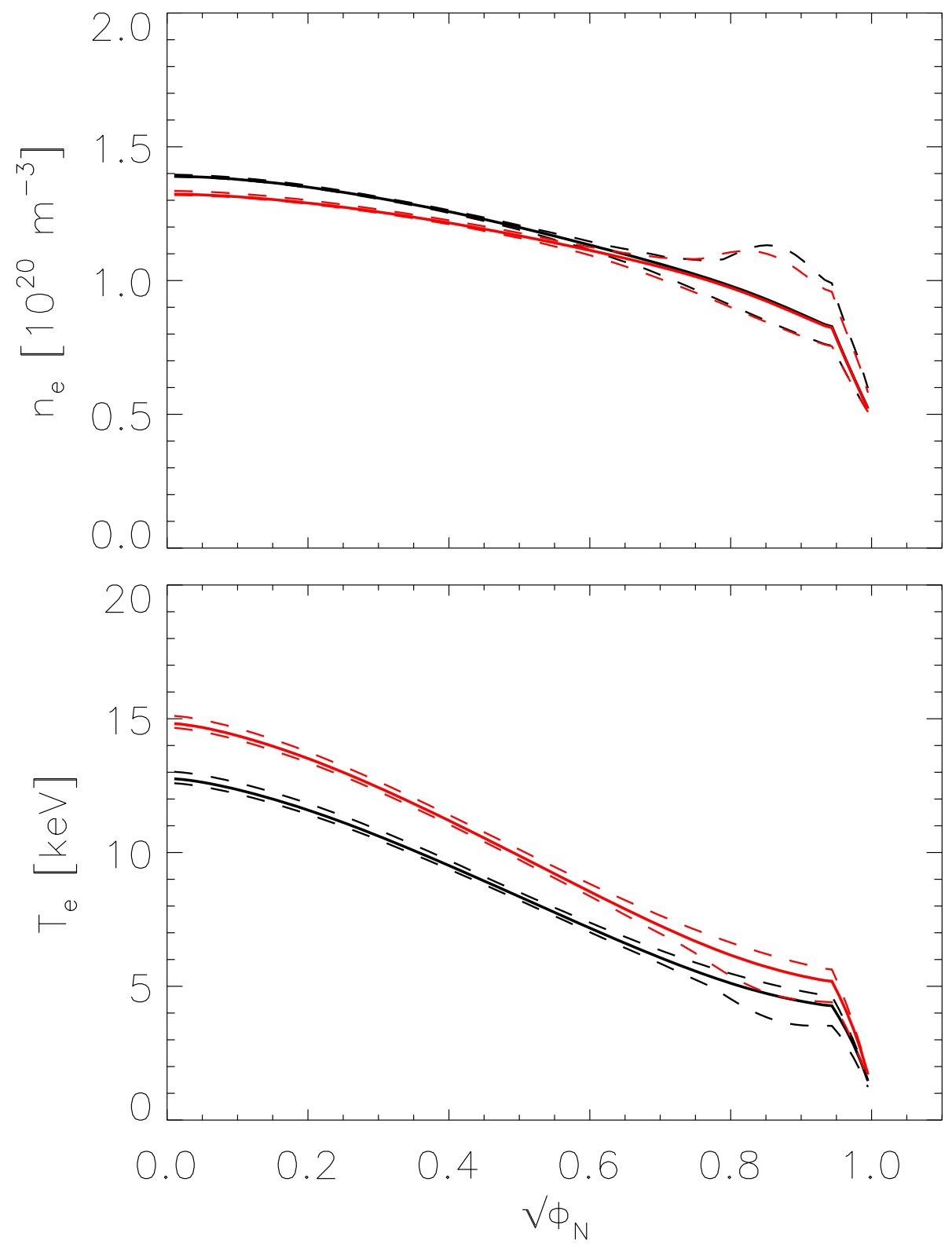

Figure 10. Same as figure 8 for the case where $\alpha_{c}$ in the continuous ELM model was reduced from 1.7 to 1.4 . 

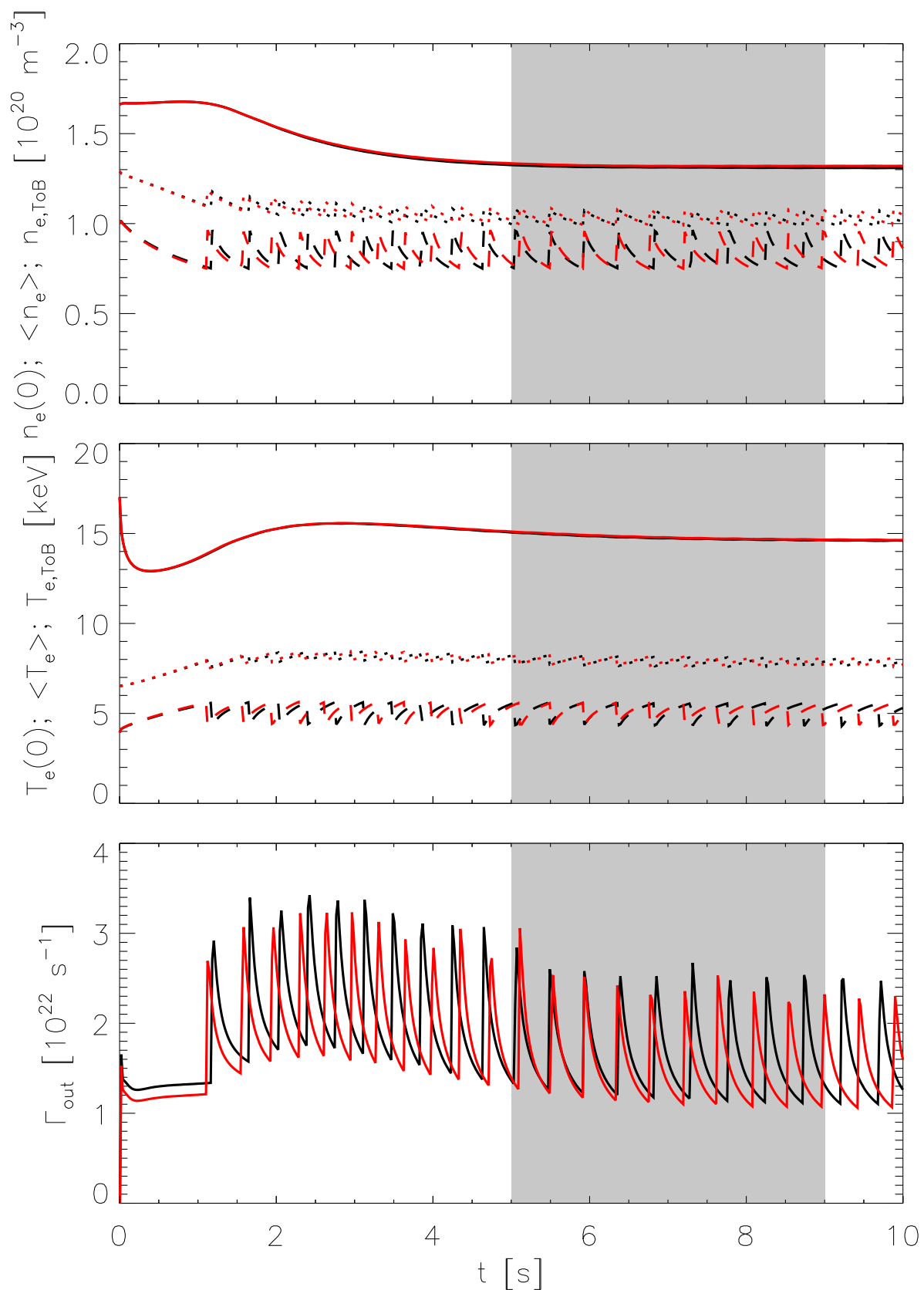

Figure 11. Same as figure 7 for the case where the recycling factor $R$ was increased from 0 to 0.2 . 

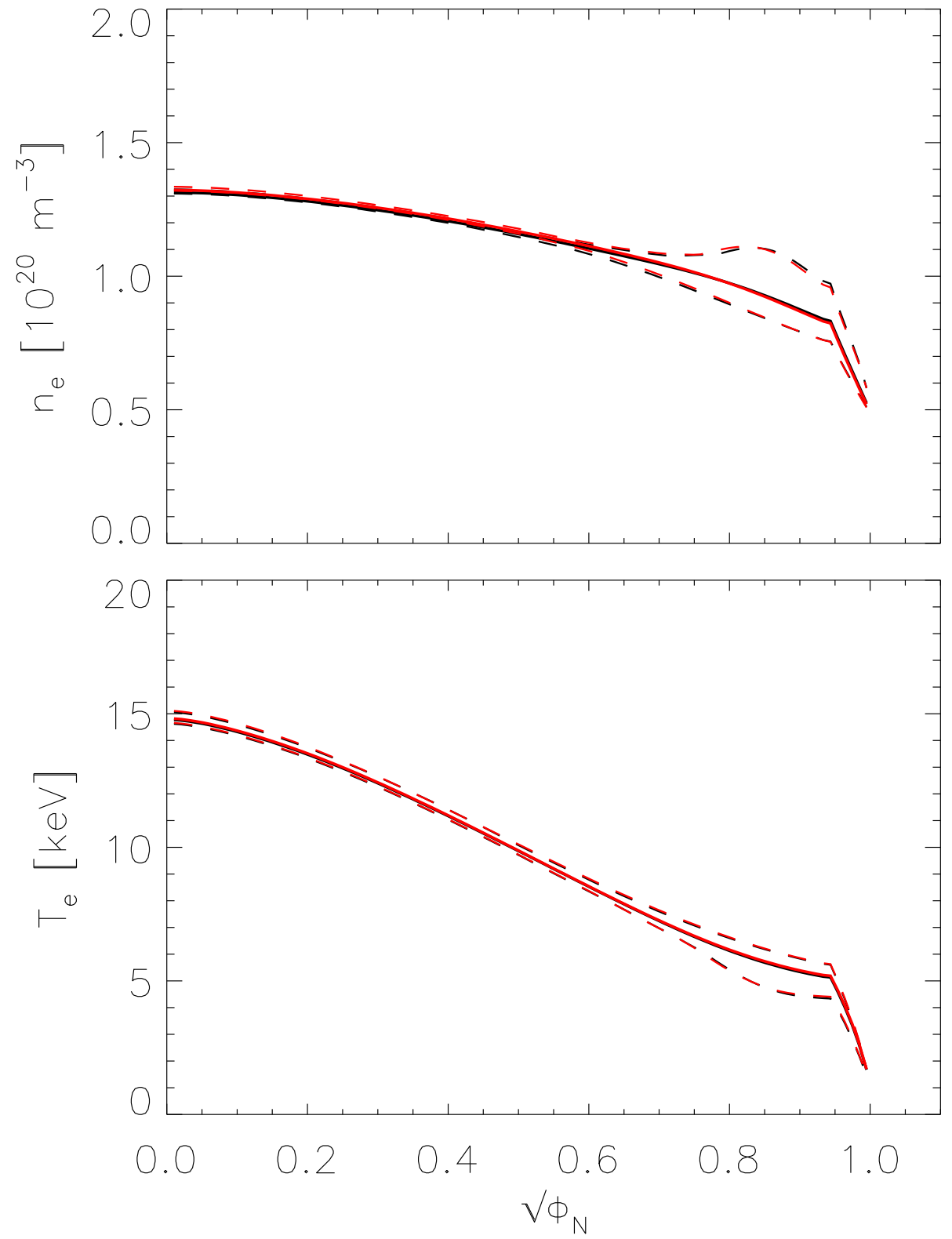

Figure 12. Same as figure 8 for the case where the recycling factor $R$ was increased from 0 to 0.2 . 

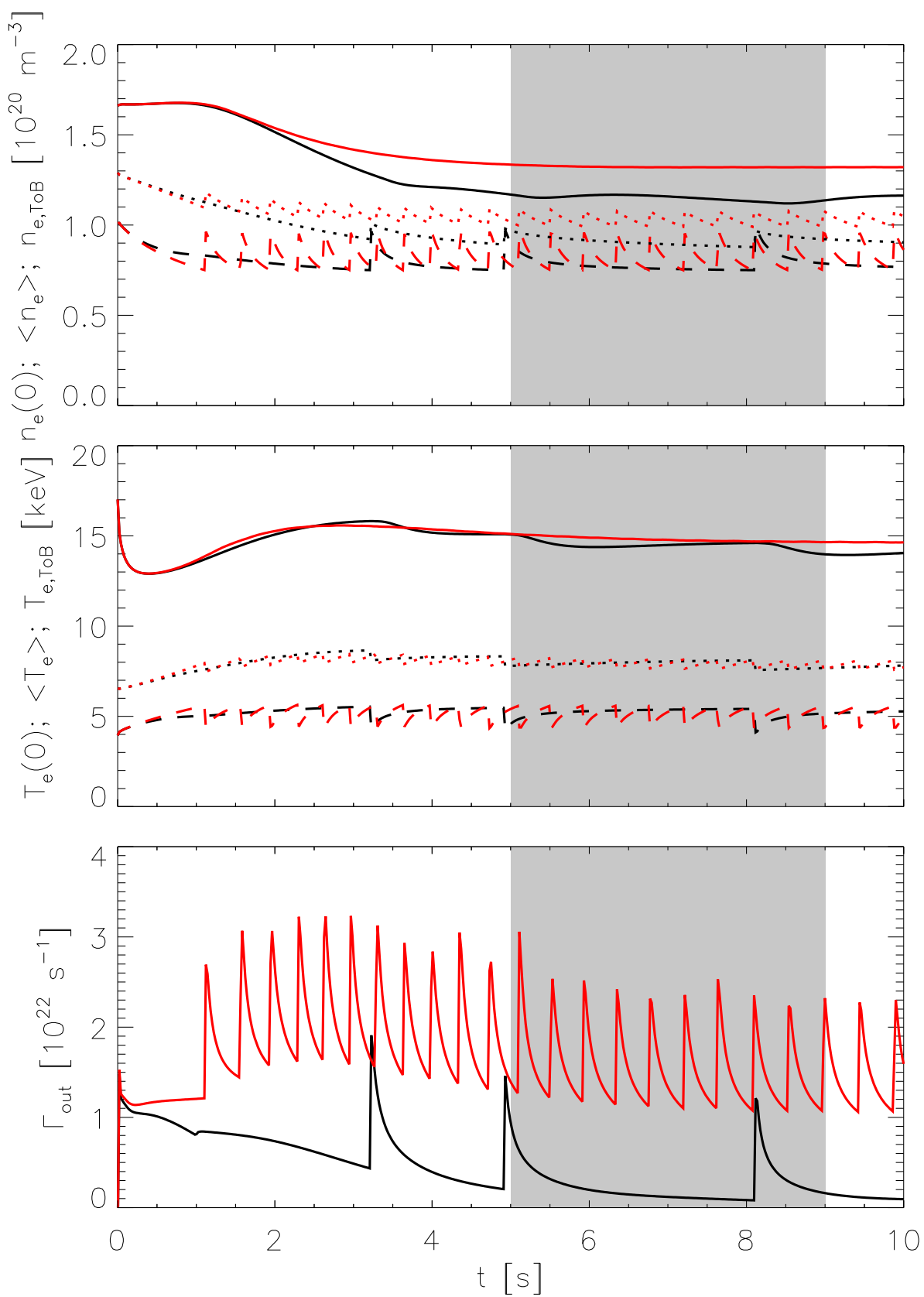

Figure 13. Same as figure 7 for the case where main ion density at the separatrix was increased from 4 to $5.3 \cdot 10^{19} \mathrm{~m}-3$ and electron and ion temperature was decreased from 800 to $500 \mathrm{eV}$. 

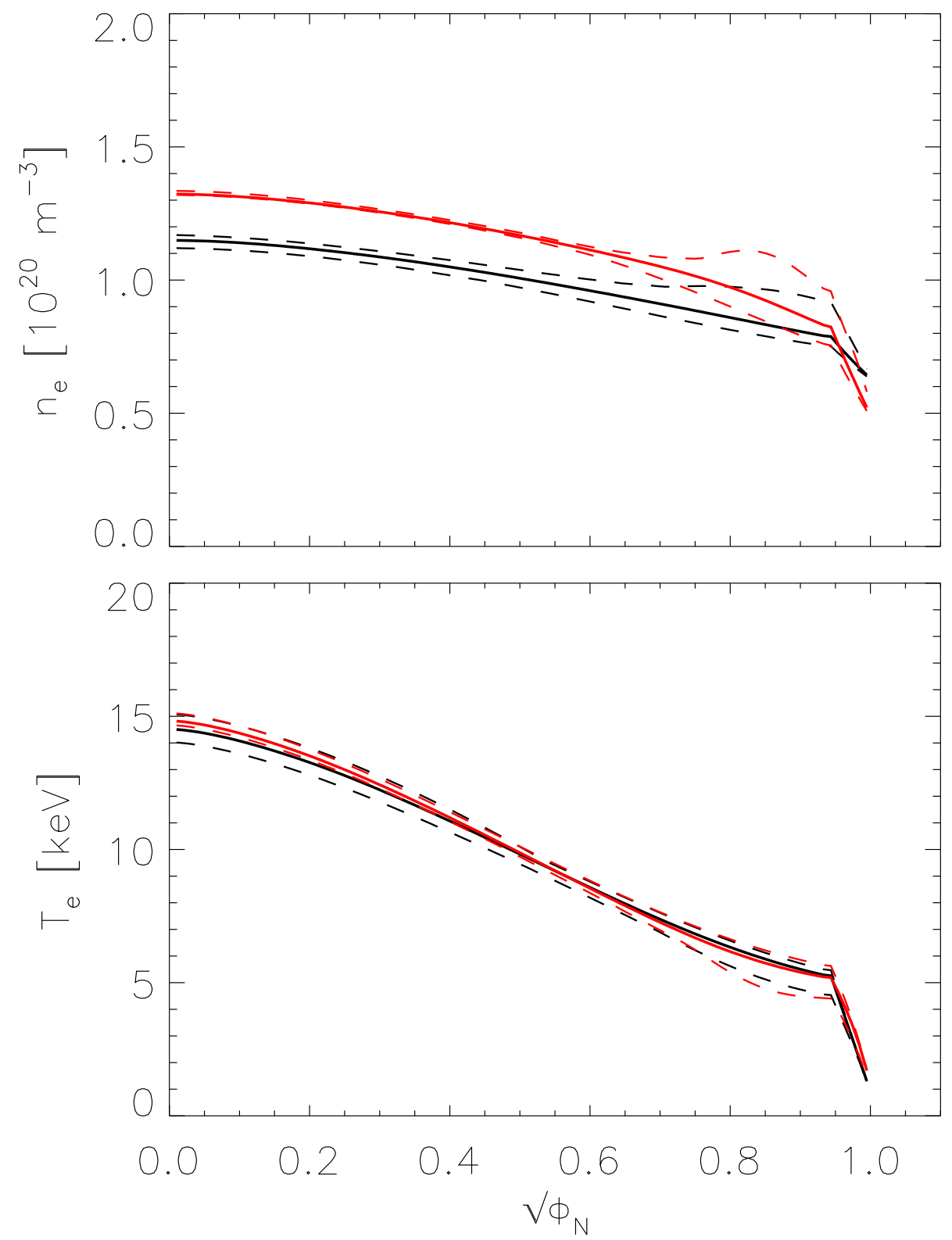

Figure 14. Same as figure 8 for the case where main ion density at the separatrix was increased from 4 to $5.3 \cdot 10^{19} \mathrm{~m}-3$ and electron and ion temperature was decreased from 800 to $500 \mathrm{eV}$. 